\title{
Stress Relaxation in SSC Collared Coils and Their Component Materials
}

F. Markley, O. Corpus, B. Herdt, A. Lautenschlager, D. Rogers, and L. Stadtler

Fermi National Accelerator Laboratory

P.O. Box 500, Batavia, Illinois 60510

March 1991

* Presented at the Third Annual International Industrial Symposium on the Super Collider [IISSC], Atlanta, Georgia, March 13-15, 1991. 


\section{Disclaimer}

This report was prepared as an account of work sponsored by an agency of the United States Government. Neither the United States Government nor any agency thereof, nor any of their employees, makes any warranty, express or implied, or assumes any legal liability or responsibility for the accuracy, completeness, or usefullness of any information, apparatus, product, or process disclosed, or represents that its use would not infringe privately owned rights. Reference herein to any specific commercial product, process, or service by trade name, trademark, manufacturer, or otherwise, does not necessarily constitute or imply its endorsement, recommendation, or favoring by the United States Government or any agency thereof. The views and opinions of authors expressed herein do not necessarily state or reflect those of the United States Government or any agency thereof. 


\title{
STRESS RELAXATION IN SSC COLLARED COILS AND THEIR COMPONENT MATERIALS
}

\author{
F. Markley, O. Corpus, B. Herdt, A. Lautenschlager, D. Rogers \\ and L. Stadtler
}

Fermilab National Accelerator Laboratory

Box 500

Batavia, IL 60510

\begin{abstract}
Previous work, reported at last year's meeting, has been continued. One model section of an SSC outer coil has had it's stress relaxation measured for nearly two years. In addition, the stress relaxation of SSC cable without insulation has been measured. Kapton insulation alone has been studied both in creep and stress relaxation. Creep has been measured parallel to the plane of the film. These measurements are dependent on internal stresses that may be present in the film and on the moisture content of the film. They indicate that any measurements of coil relaxation are not likely to be repeatable if these factors are not controlled.

The measurements on Kapton film alone are being done at different temperatures to obtain a time-temperature shift factor so that measurements at one temperature may be used to predict relaxation at any other temperature.
\end{abstract}

\section{INTRODUCTION}

At the last IISSC, we presented data on the stress relaxation of short sections of SSC outer coil. In order to better understand this data, we have been measuring the relaxation properties of the individual coil components. These measurements are being made in order to understand how the compressive stress in collared coils decays with time, especially during storage of magnets at ambient temperatures or even at higher than ambient temperatures in an uncooled warehouse prior to installation. The goal is to be able to predict the time-temperature history acceptable to maintaining enough compressive stress to prevent coil motion during operation and the quench that might result. Since the Kapton insulation is a thermoplastic it is reasonable to expect it to undergo the greatest relaxation. However it is important to keep in mind that the copper (and superconductor) make up the greatest part of the coil volume, and the true stress at the strand-to-strand contact points must be equal to the yield stress. The Kapton sees the same maximum stress of course, but each high stress point is surrounded by low stressed supporting material which is not true for strand. The epoxy-fiberglass should relax less than the Kapton since the glass should not relax much at all and the epoxy is a cross-linked plastic that is expected to undergo an initial relaxation but then stop.

Measuring the relaxation rate of Kapton film in the direction perpendicular to the plane of the film is very difficult, but that is the direction of the stress in SSC coils. 
Measuring a single thickness of .001 inch film lacks sensitivity and is strongly influenced by friction effects with the load applying members. Measuring a stack of many layers of film introduces a large error in knowing the actual thickness of the stack. We therefore decided to do two measurements. One would be the perpendicular measurement using 100 layers of .005 inch thick film. The other would be a measurement in the plane of the film, and would be a measurement of tensile creep. We felt that this would be the most accurate measurement of the viscous-elastic properties of Kapton, and therefore would be the best method to use at two different temperatures in order to get the time-temperature shift factor for Kapton. The shift factor should be relatively independent of the stress direction and whether stress or strain were held constant during the measurement.

\section{KAPTON CREEP MEASUREMENT}

Figure 1 shows the experimental design for the creep experiment. We used a double strip of .001 inch Kapton .375 inches wide and $\mathbf{1 5 . 3 5}$ inches long. The core of a linear variable differential transformer was fastened to the lower end to read the extension. A weight was attached to the other end of the core. The transformer coils were supported by a tube rolled from Kapton sheet and epoxied together. This arrangement cancelled out any thermal expansion effects. The whole apparatus was enclosed in a double walled acrylic tube with circulating water in the space between the walls for temperature control. The water was supplied by a commercial water bath with both heating and cooling modes. We made several measurements with this apparatus with completely inconsistent results. We then became concerned about the effect of moisture absorbed into the film. Dupont reports that at $100 \%$ humidity Kapton can absorb up to $3 \%$ it weight in water. This absorbed water content would change with changes in humidity and temperature causing dimensional changes larger than the viscoelastic effect. It is also true that the Kapton film can maintain internal stresses which are released by temperature causing additional dimensional changes. These effects mask any relaxation phenomena unless they are controlled by heating the film to dry and anneal it prior to testing. Of course dryness must be maintained during the measurement. A fixed humidity other than $0 \%$ could be used, but with increased experimental complexity. We think that the variability of our stress relaxation data on coil sections reported last year may be due lack of control of these factors. Variability of Kapton humidity and internal stresses during manufacture can also be expected to change stress levels and future behavior. The .001 inch film thickness used in these creep measurements allowed rapid diffusion of water into and out of the film to cause the dramatic changes we observed. We now oven dry and anneal the Kapton prior to a measurement and we maintain $0 \%$ humidity during the test with a drying agent in the acrylic chamber. Figure 2 is a graph of the first creep data obtained with these new controls. The data were taken at a temperature of $130^{\circ} \mathrm{F}$, which could easily be reached in a hot warehouse in summer. This test is still ongoing, and we have not yet tried to fit an analytical expression to the data. We still have to measure creep at a different temperature to get the time-temperature shift factor.

\section{KAPTON STRESS RELAXATION}

The stress relaxation measurements on a stack of Kapton have benefited from the problems in the creep measurement and we carefully dry and anneal the film before stacking it. Again we use drying agent in the temperature controlled enclosure. Figure 3 shows the apparatus for the film stress relaxation experiment. The $.5 x .5 \times .5$ inch cube of Kapton consists of 100 layers and is compressed by bolting the top onto the $U$ shaped steel fixture. The Kapton is held between the top of the fixture and a movable steel plate resting on a commercial load cell. The load cell is excited with a constant current supply and both the input voltage and output voltage are periodically measured and recorded to compute the load on the Kapton. The entire assembly is kept in an acrylic box with a heater and fan and some drying agent. We have two identical setups and run both simultaneously. Figures 4 and 5 present the first set of data from these fixtures. Figure 4 was a $130^{\circ} \mathrm{F}-100^{\circ} \mathrm{F}$ $130^{\circ} \mathrm{F}$ run and figure 5 was a $100^{\circ} \mathrm{F}-130^{\circ} \mathrm{F}-100^{\circ} \mathrm{F}$ run in the other fixture. Since we have not 


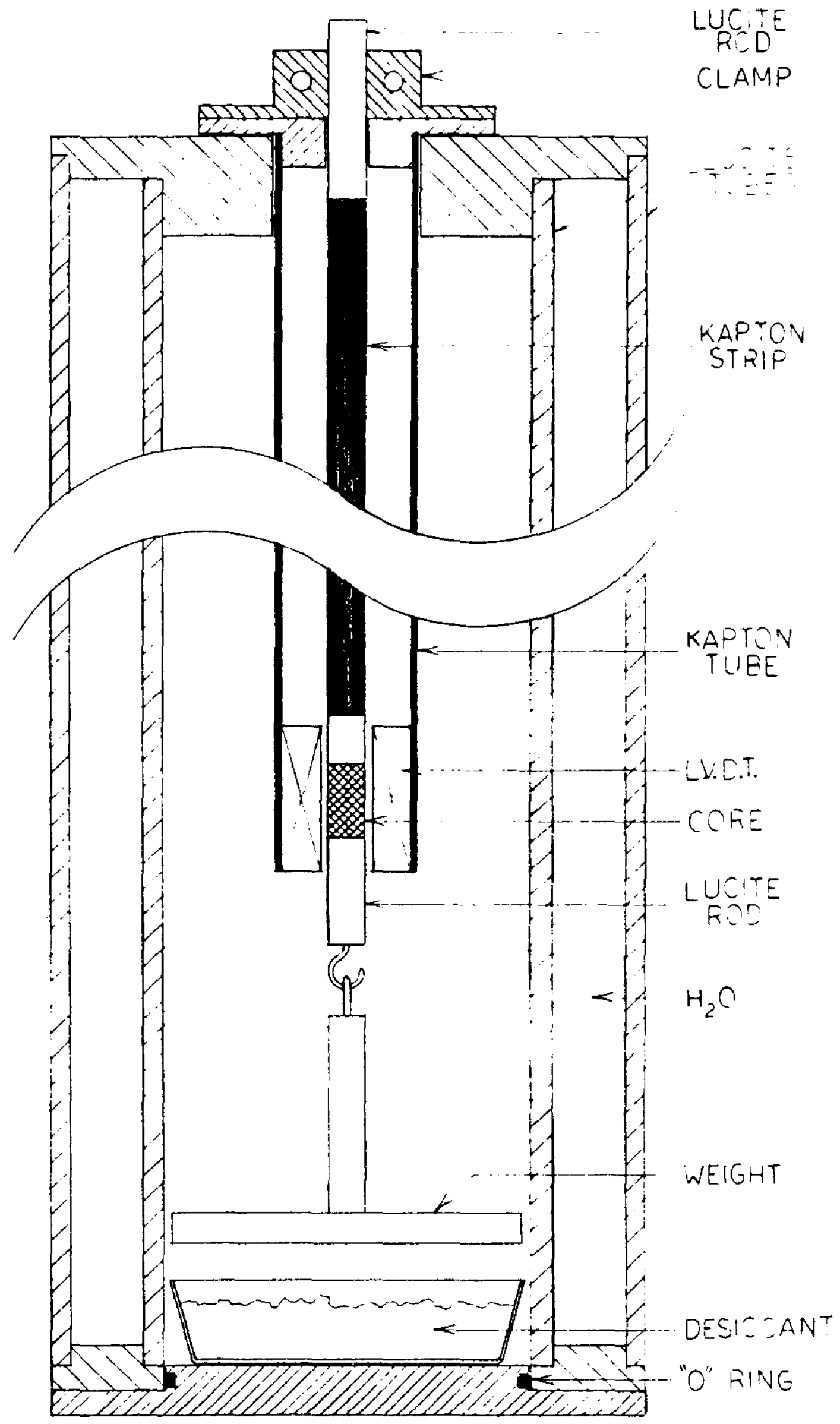

Figure 1. Kapton creep Apparatus 


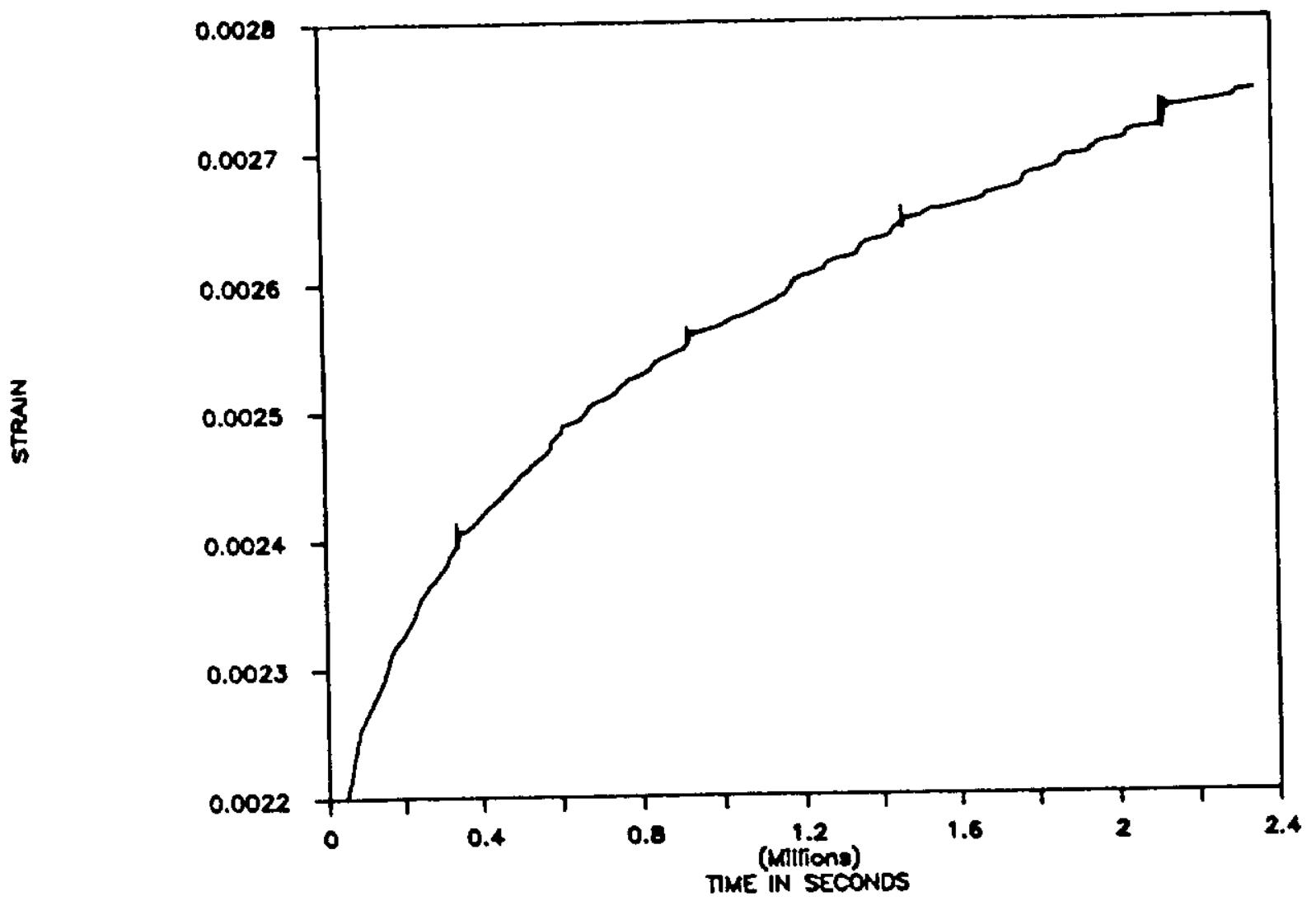

Figure 2. Kapton creep

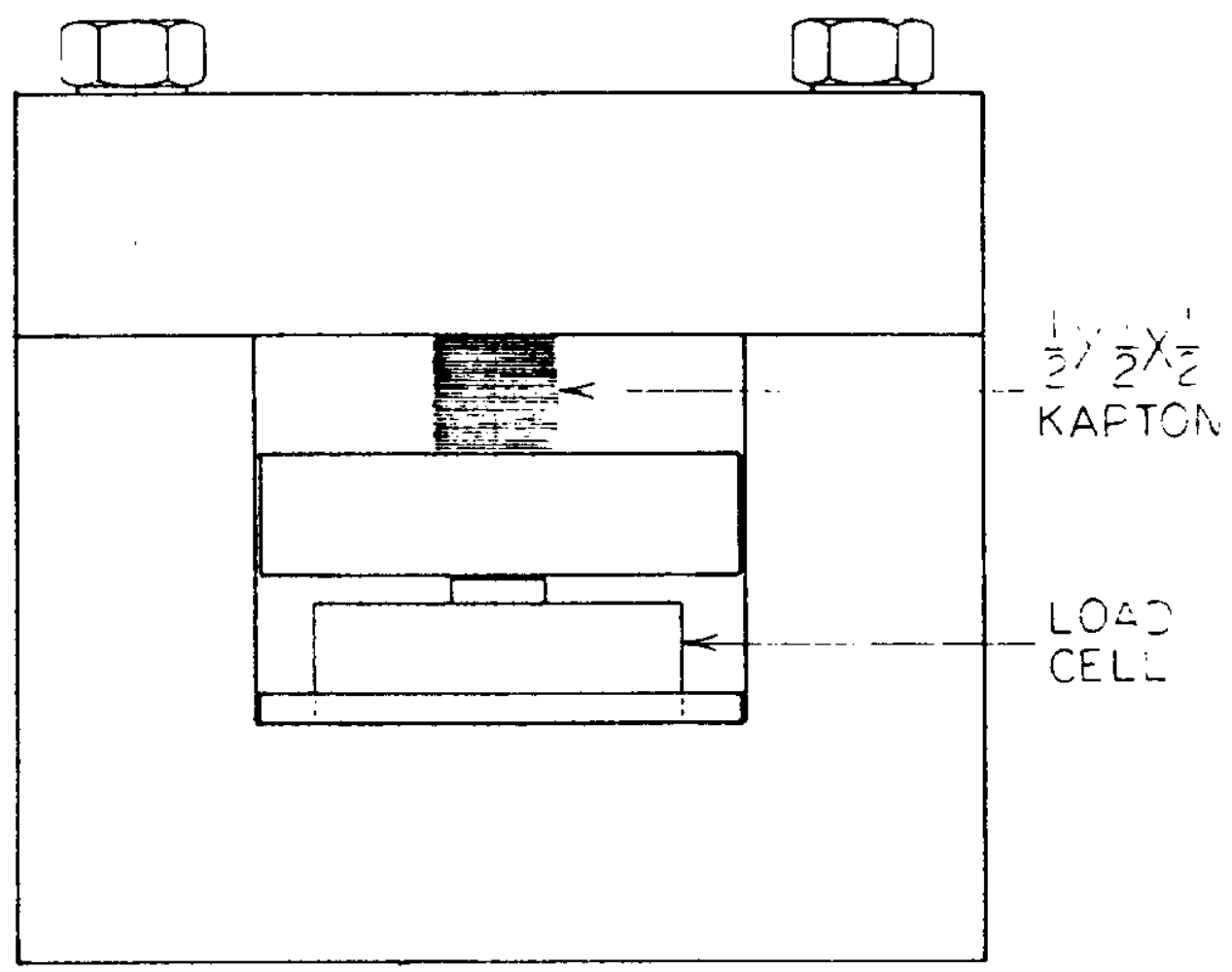

Figure 3. Kapton stress relaxation fixture 
gotten two temperature runs on the creep experiment, we have attempted to analyze this stress relaxation data to get the time-temperature shift data. We can get around the uncertainty in the exact stack height by using the different temperature data on the exact same sample with no other changes.

As we did with the coil stress relaxation data presented last year, we have tried to fit the data with an expression of the form $l=10-11 \mathrm{xt}^{\mathrm{n}}$ where 10 is the time independent load and $11 t^{n}$ is the time dependent load chosen because other workers have found a lot of creep data to be well fit with a logarithmic expression. Obviously the expression can be written using either load or the relaxation modulus as a function of a time independent term and a time dependent term. We fit the equation to the first $1 / 3 \mathrm{rd}$ of the data to find the parameters 10,11 , and $n$. Then using the superposition principal we find a value of the time-

temperature shift factor which allows the same parameters to best fit the remaining $2 / 3$ rds of the data. To fit the curve to the first $1 / 3$ rd we have found it convenient to select 3 points on a smoothed curve to be used in writing three simultaneous equations to solve for 10,11 , and $\mathrm{n}$. We can algebraically eliminate 10 and 11 to get an equation in $n$ alone which can be graphically solved by plotting the value of the equation versus $n$ and locating the zero crossing. We can then calculate 10 , and 11 from $n$. To fit the remaining $2 / 3$ rds of the data we assume superposition of three different loads. The first load is 10 and is applied at $t=0$. The second load is applied at $t=t 1$ and is the result of the difference in thermal expansion of Kapton and fixture when the temperature is changed. The third load is applied at $t=t 2$ and is the negative of the second load and occurs on changing the temperature back to its first value. The first load must be followed thru 3 different temperature regimes, and the second load must be followed thru 2. When temperature is changed the time scale changes by a factor of $k$. Thus we get for the middle (hot) section that the load is the sum of the first or l0$11(t 1+k(t-t 1))^{n}$ and the second or dlo-dll(k(t-t1) $)^{n}$. dlo is the increased load due to the greater expansion of the Kapton. We can find dl 1 if we assume that $10 / 11=\mathrm{dl} 0 / \mathrm{dl} 1$. For the third section we must sum three terms. Thus we get that the load is the sum of la=10$11(t 1+k(t 2-t 1)+(t-t 2))^{n}$ plus $l b=d l 0-d l 1(k(t 2-t 1)+(t-t 2))^{n}$ plus $l c=-d l o+d l i(t-t 2)^{n}$. For the data with the cold middle section, if we fit the first section with lo-l1 $(t)^{n}$, then the middle section is represented by $l=10-11\left((t 1+(1 / k)(t-t 1))^{n}-d l 0+d l 1\left((1 / k)(t-t 1)^{n}\right.\right.$, and the third section is represented by $\mathrm{l}=10-11(\mathrm{t} 1+(1 / \mathrm{k})(\mathrm{t} 2-\mathrm{t} 1)+(\mathrm{t}-\mathrm{t} 2))^{\mathrm{n}}-\mathrm{dl} 0+\mathrm{dl} 1((1 / \mathrm{k})(\mathrm{t} 2-\mathrm{t} 1)+(\mathrm{t}-\mathrm{t} 2))^{\mathrm{n}}+\mathrm{d} 10-\mathrm{dl} 1(\mathrm{t}-\mathrm{t} 2)^{\mathrm{n}}$

Since we do not yet have a good figure for the coefficient of thermal expansion of Kapton we took the liberty of adjusting $\mathrm{dlO}$ (within a reasonable range) as well as $\mathbf{k}$ in order to get the best fit to the data. We use dlo $=150 \mathrm{lbs}$ for the figure 4 data and dl0 $=135 \mathrm{lbs}$ for the figure 5 data which are probably consistent within fixture dimensional variation. The data shown in figures 4 and 5 each gives us one determination of 10,11 and $n$, and two determinations of $k$. These are not very accurate determinations, but the best values so far are $n=.05$ and $k$ ranging somewhere between 2 from figure 4 data and 25 from figure 5 data. These must be considered preliminary data to be refined as our measurements continue. In fact we are noticing that some of our Kapton data begins to look quite linear after some time and therefore could be fit using an exponential plus a linear function instead of the logarithmic one. Linear behavior would be expected if there is a strictly viscous component. Thermoplastic materials like Kapton usually have such a component.

\section{BARE CABLE STRESS RELAXATION}

In order to examine the stress relaxation of SSC cable without any insulation, we measured an SSC outer coil section just like we used last year except that all the insulation was removed and replaced by a steel shim of equal thickness at the parting plane. Actually 4 inch sections of cable were used, but they were loaded only over a 3 inch length. This made it possible to tape the cable ends to prevent them from unwinding during handling. The data is given in Figure 6 as curve b4, along with the data on insulated coil sections presented last year. The curve marked B4 is the bare cable data. All the curves have been normalized to start at 1 by dividing all the points by the value of the first point on that curve. It is surprising to note that the bare cable stress relaxation accounts for a substantial part of the total coil relaxation. Figure 7 shows

the bare cable relaxation over a much larger time. The rate of relaxation appears to steadily 


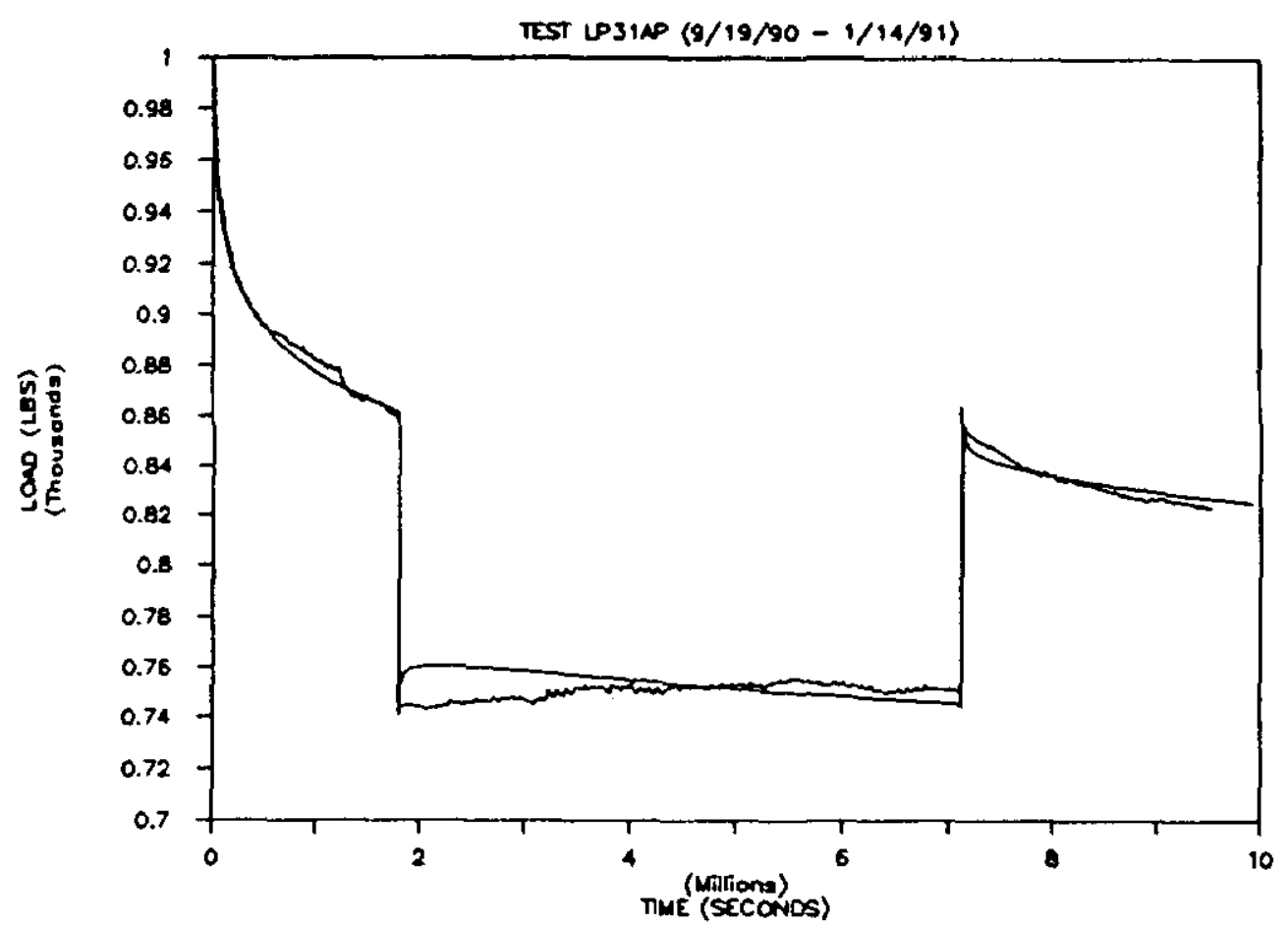

Figure 4. Kapton compressive stress relaxation

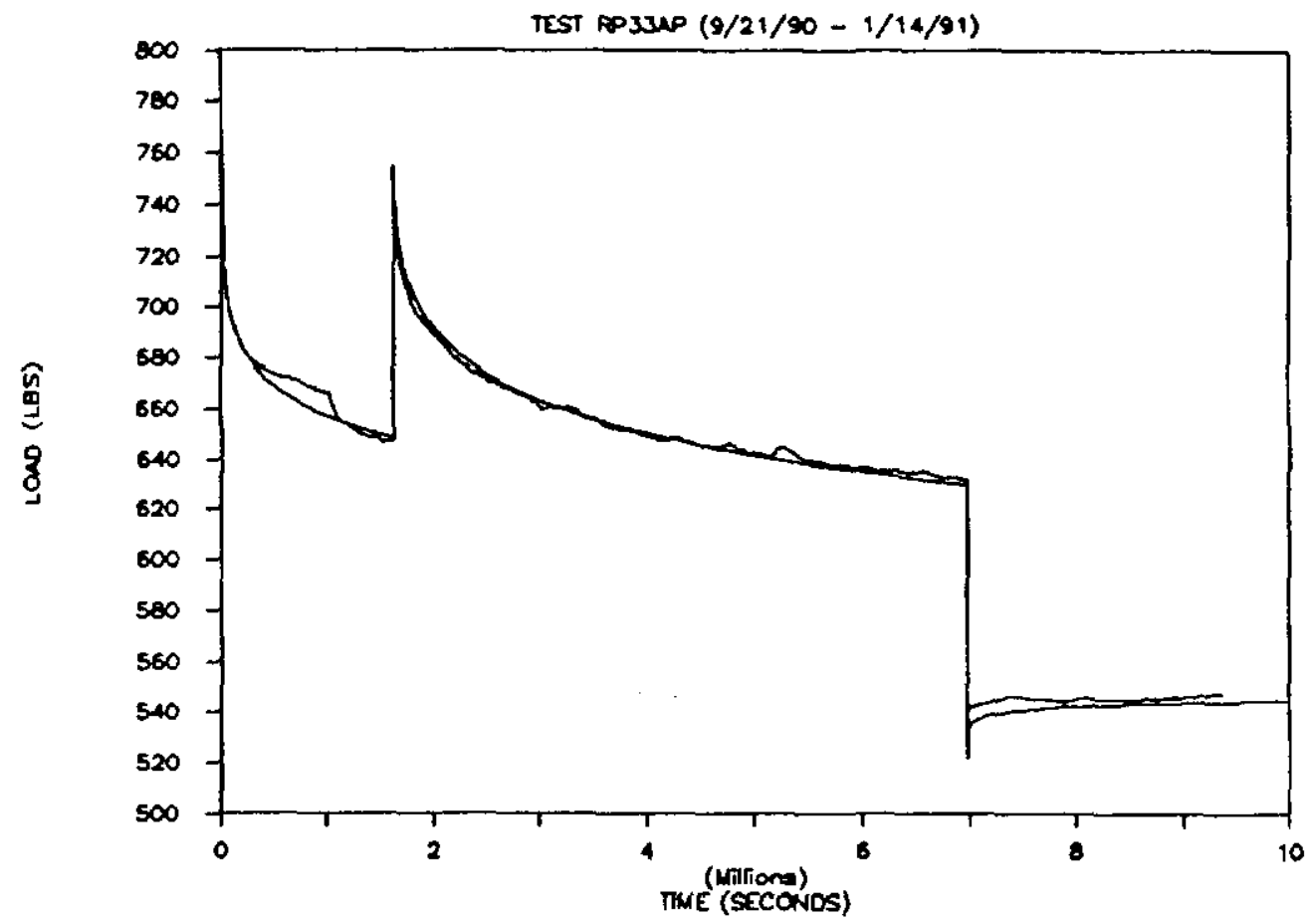

Figure 5. Kapton compressive stress relaxation 


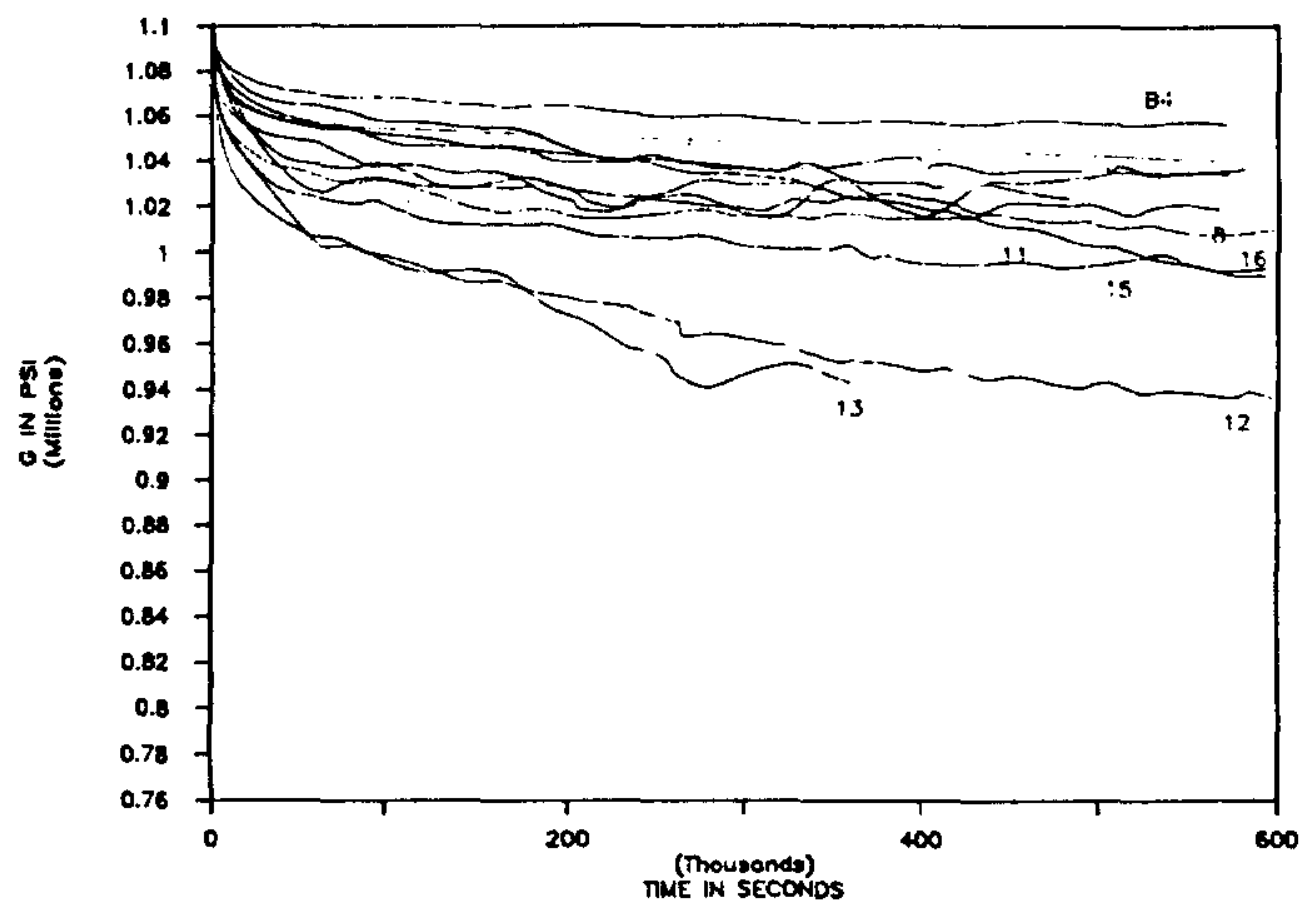

Figure 6. Normalized data

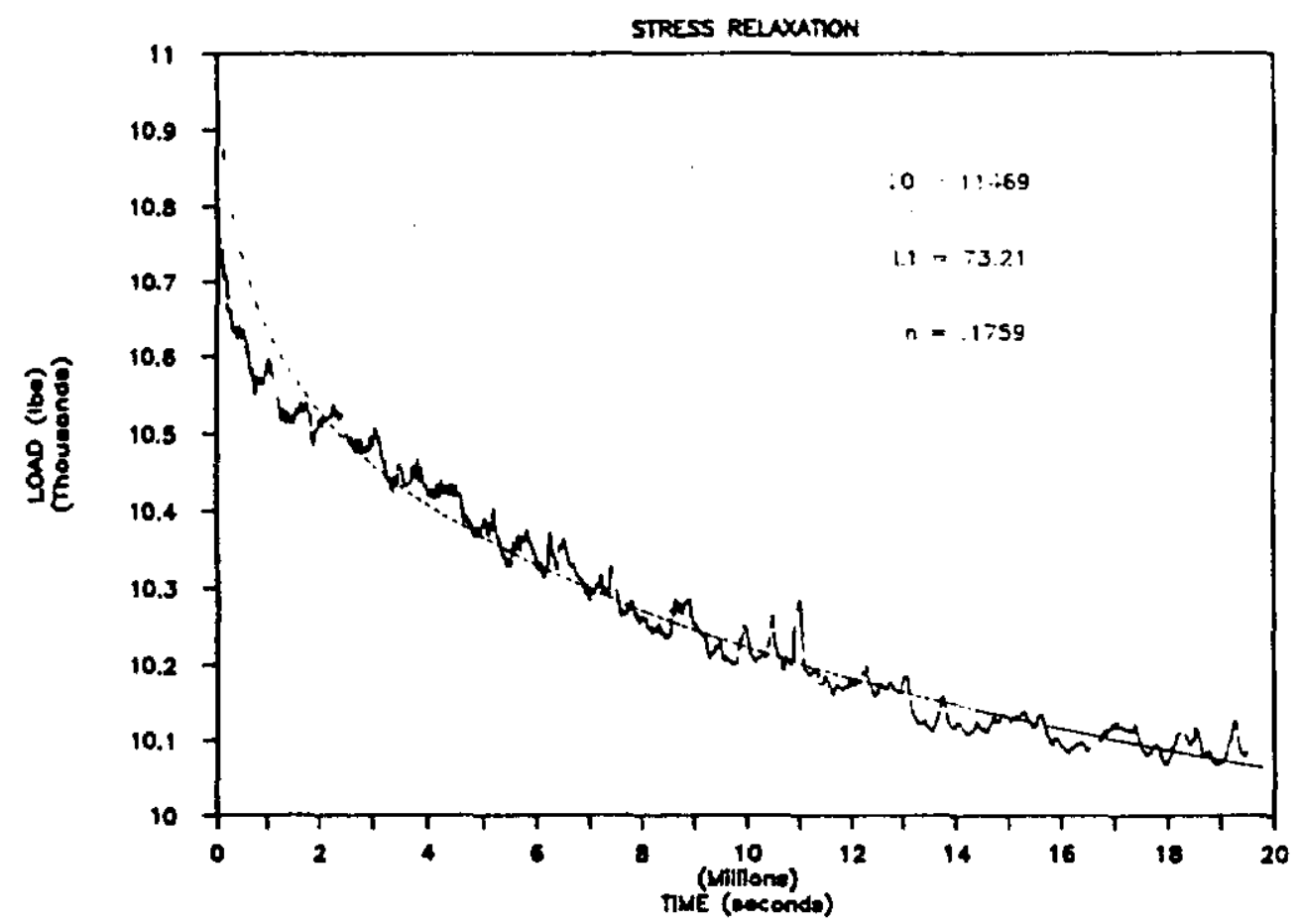

Figure 7. "Bare Wire" coil sample *B4 


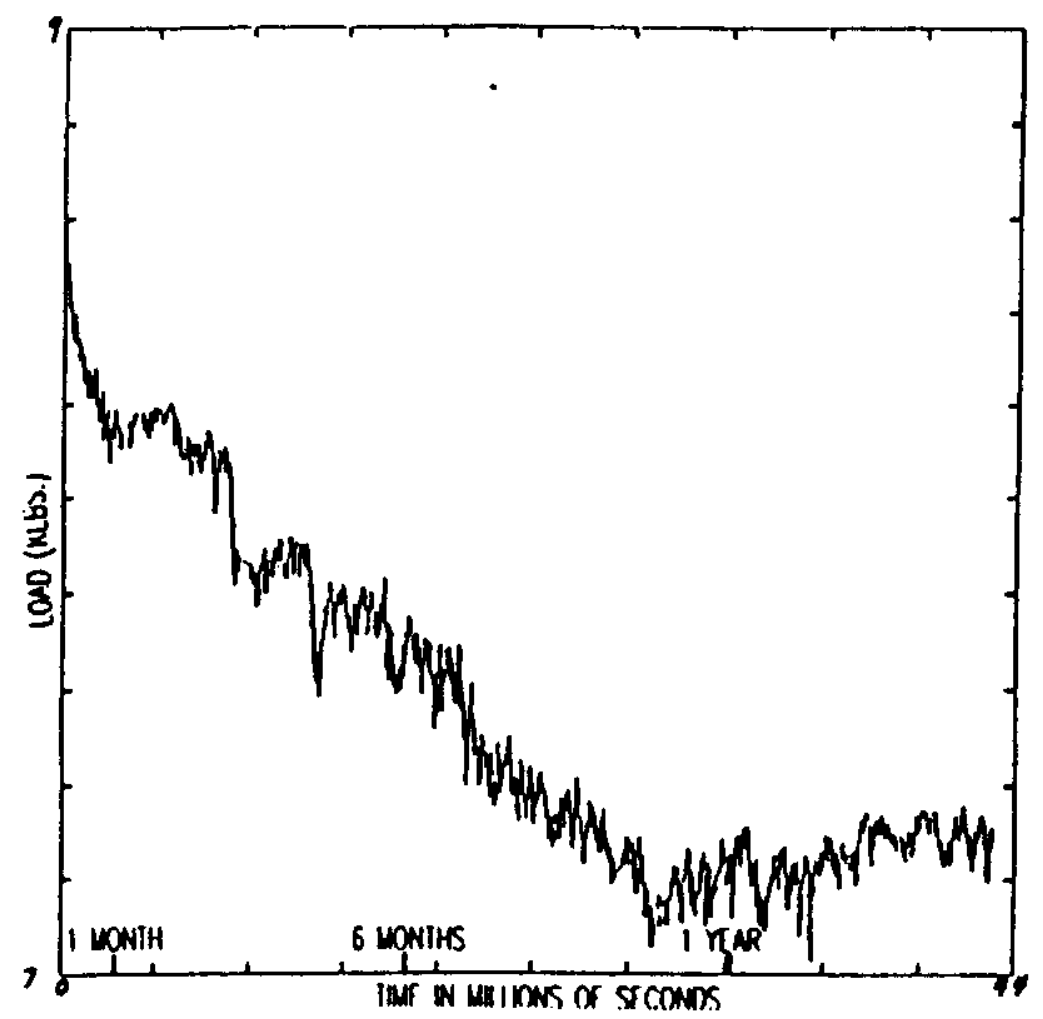

Figure 8. "Long Term" stress relaxation test (coil sample \#15)

decrease. If we fit the $l=10-11 \mathrm{xt}^{\mathrm{n}}$ expression to the data we get values of $10=11469,11=73$, and $\mathrm{n}=.18$. Note that this is the result of a single measurement.

\section{ADJUSTING FOR DIFFERENT TEMPERATURES}

If we assume that the time-temperature coefficient has the form $\ln \mathrm{k}=\mathrm{Q}((1 / \mathrm{T} 0)$ (1/T1)) where lnk is the natural logarithm of $k$, our measured shift factor, and T0 is a reference temperature, and $\mathrm{T} 1$ is a higher temperature, and $\mathrm{Q}$ is some constant, then we can calculate $Q$ and use it to find a shift factor $k$ for any two temperatures. The hot and cold temperatures we used in the measurement were 130 degrees $F$ and 100 degrees $F$.

Converting to degrees Kelvin and calculating the difference we find that $Q=6130 x \ln$. The value of $\mathbf{k}$ we found by fitting the cold-hot-cold and the hot-cold-hot stress relaxation curves of Figures 4 and 5 was was somewhere between 2 for the former and 25 for the latter. In an earlier paper ${ }^{2}$, we measured the $Q$ value for a Kapton and epoxy-fiberglass insulated straight 10 stack of Tevatron cable and got a value of 11400 . That number would translate to a k value of 6 for our present 100 to 130 degree $F$ temperature range. Even though we are comparing a complete coil with straight Kapton it is true that we expect the most temperature sensitive element in the coil to be its Kapton insulation and therefore the time-temperature shift should be similar. The value of $n$ found in fitting a curve of the $l=10-11 \times t^{n}$ type to the Tevatron coil data was .08 while we here report .05 for Kapton alone and .18 for bare cables. These values indicate that bare cable, using the average compressive stress, not the true 
strand stress, relaxes faster than Kapton using a real stress value equal to the cable average stress value.

\section{COIL RELAXATION}

Figure 8 shows the continued relaxation of the SSC outer coil section reported last year. This section has now been measured for $\mathbf{4 3}$ million seconds, but the slope starts to increase at about 27 million seconds which is probably due to some problem in the apparatus. With this fixture we have not yet begun to record both input and output voltages to the load cell so it is possible that our supply of voltage has gone awry.

\section{FUTURE PLANS}

A major goal is to reduce the order of magnitude uncertainty in the time temperature shift factor $k$. We are planning to measure the elastic modulus of dry annealed Kapton perpendicular to the plane of the film at liquid helium temperatures. If we assume that viscoelastic processes are frozen out at that temperature, we will have a direct measurement of the time independent portion of the viscoelastic behavior. We have also started a careful measurement of the thermal expansion properties of dry annealed Kapton perpendicular to the plane of the film. Thus we can eliminate one variable in the determination of the time-temperature shift factor $k$ as discussed in the section on Kapton stress relaxation. We are also carry out several different measurements of the total stack height of the 100 layer stacks used in the Kapton stress relaxation experiments. Obviously, additional measurements are needed to assure repeatability, especially with the bare cable. We will continue to look for the best curve fit to the data so that extrapolations to long times can be made with some confidence, at least for dry annealed Kapton and bare cable.

1. Markley , F.W. and J.S.Kerby, "Investigation of the Mechanical Properties of Superconducting Coils," Supercollider 2. Vol. 2, Plenum Press, New York, 1990, pp 753 . 763.

2. Carson, J.A., and F.W.Markley, "Mechanical Properties of Superconducting Coils." IEEE Transactions on Magnetics, Vol. Mag. 21 \#2, March 1985, pp. 85. 